\title{
Modeling, Simulation, Docking Studies of Tyrosine Kinase Involved in Leukemia and Microwave Synthesis of Quinazoline Derivatives
}

\author{
${ }^{1}$ Raju Bathula, ${ }^{*}$ Satla Shobha Rani, ${ }^{2}$ K. Rama Devi \\ ${ }^{I}$ Centre for Pharmaceutical Sciences, IST, JNTUH, Kukatpally, HYD-85. India. \\ ${ }^{2}$ Department of Pharmacy, Anurag Group Of Institutions, Venkatapur, Ghatkesar, Hyderabad. \\ Corresponding author: \\ Dr.S. SHOBHA RANI, Head Of The Department, \\ Centre for Pharmaceutical Sciences, Institute of Science and Technology, JNTUH, Kukatpally, HYD-85. India. \\ Mail: satla_shobharani@yahoo.com
}

\begin{abstract}
Acute Mylenoid Leukemia is a type of cancer which affects blood and bone marrow and is characterized by an abnormal proliferation of white blood cells. A number of genes are involved in regulating heamatopoiesis, and thereby affecting the susceptibility to Leukemia.One among them Tyrosine kinase which plays an important role in cell proliferation and differentiation. In this work three dimensional model of Tyrosine kinase was generated using 1Z3S as a template with the help of Modeller7v7. With the aid of the molecular mechanics and molecular dynamics methods, the final model is obtained and is further assessed by Procheck and Verify $3 D$ graph programs, which showed that the final refined model is reliable. After energy minimization the three dimensional structure of Tyrosine was compared with template. Molecular docking of 6thiogunaosine (6-TG) analogs was performed on the ATRI model and selective inhibitor was selected based on the docking results. The docking results showed that the three residues in the Tyrosine kinase (GSN235, AsN257, and ILE289) were essential for making hydrogen bond with the analogues. The data presented here strongly indicate that the interactions of these four residues are necessary for a stronger binding of the Tyrosine kinase with synthesized molecules. Also, the study proposed analogues were an effecctive inhibitors by the comparison of docking energy. Synthesis is of Structure Of 3'-(4-Oxo-2-Phenylquinazolin-3(4h)-Yl)-4'hSpiro[Indole-3,2'-[1,3]Thiazolidine]-2,4'(1h)-Dione derivatives were not possible because of stearic hindrance by bulky groups present on the nitrogen atoms.
\end{abstract}

Keywords: Acute mylenoid Leukemia, Tyrosine Kinase, Modelling, Molecular Dynamics, Docking studies, stearic hindrance.

\section{Introduction}

Tyrosine Kinase is one of the most mutated gene in the human leukemia, is a class III receptor tyrosine kinase (RTK) protein that is an important regulatory gene involved in normal heamatopoiesis (Gillaland DG, Griffin JD 2002 and Small D. 2006). RTK also includes C-KIT, C-FMS, platelet derived growth factor (PDGF) and membrane bound receptor with intrinsic tyrosine kinase domain. FLT3 is a share common structure with class III RTKs consisting of 5 extracellular immunoglobulin domain, single transmembrane domain, a cytoplasmic juxtramembrane region, and highly conserved a cytoplasmic kinase domain interrupted by a kinase insert. Tyrosine kinase recepotor exists as monomeric, unphosphorylated state with an inactive kinase moiety in the unstimulated state. After the interaction of the receptor with FLT ligand (FL), receptor undergoes conformational change, which results in the unfolding and the exposure of the dimerization domain, allowing receptor-receptor dimerization. The receptor dimerization is important to the activation of the tyrosine kinase enzyme which leads to phosphorylation of various sites in the intracellular domain. In the Intracellular domain, the activated receptor along with many proteins in the cytoplasm forms a complex of protein-protein interactions. FL activates the different downstream targets, which include proteins in the activators of transcription (STAT), signal transducers, mitogen activated protein (MAP) kinase, and AKT pathways that are involved in the regulating, proliferation, differentiation and cell survival. In vitro studies have shown that the activated Tyrosine Kinase controls downstream signaling pathways which results in continuous cellular proliferation and resistance to apoptotic cell death. Activation of Tyrosine kinase occurs through two main mechanisms: coexpression of FL, which leads to activation of autocrine, paracrine, or intracrine signaling and via conferring ligand independence, mutation of the FLT3 gene itself (Abu-Duhier FM et al 2001, Drexler HG (1996), Meierhoff G et al 1995, Nakao M et al 1996). These mutations are internal tandem duplications of the juxtramembrane domain (ITD), or point mutations of the second tyrosine kinase domain (TKD) and point mutations of the juxtramembrane domain. Constitutively activated FLT3 contributes to the leukemic phenotype 
in transgenic murine model systems (Grundler $\mathrm{R}$ et al 2005, Li L et al 2008). FLT3 primarily expressed in early lymphoid progenitors and myeloid plays an important role in proliferation and differentiation. FLT3 expression has been described in lymphoheamtopoitic organs such as the liver, spleen, thymus and placenta. The majority of human acute leukemias, including $100 \%$ of B-cell lineage acute lymphoblastic leukemias (ALL), $27 \%$ of Tlineage ALL, and 89\% of acute myelogenous leukemias (AML) overexpress Tyrosine Kinase (Brig F et al 1992, Carow CE et al 1996). ITD mutations are found in 3\% of patients with myelodysplastic syndromes (MDS) (Shih LY et al 2004), and up to $15 \%$ and $25 \%$ of pediatric and adult AML patients, respectively. In both pediatric and adult AML patients, the presence of an ITD mutation is associated with a significantly higher relapse rate and worse overall survival (Klo H et al 1999 and Meshinchi S et al 2003).

\section{D model building}

\section{Methods}

The initial model of Human Tyrosine Kinase protein was built by using homology-modeling methods and the MODELLER software; a program for comparative protein structure modeling optimally satisfying spatial restraints derived from the alignment and expressed as probability density functions (pdfs) for the features restrained. The pdfs restrain $\mathrm{C}^{\alpha}-\mathrm{C}^{\alpha}$ distances, main-chain $\mathrm{N}-\mathrm{O}$ distances, main-chain and side-chain dihedral angles. The 3D model of a protein is obtained by optimization of the molecular pdf such that the model violates the input restraints as little as possible. The molecular pdf is derived as a combination of pdfs restraining individual spatial features of the whole molecule. The optimization procedure is a variable target function method that applies the conjugate gradients algorithm to positions of all non-hydrogen atoms.

\section{Domain Identification and Template Search}

The query sequence from Homo sapiens was submitted to domain fishing server for Human Tyrosine Kinase protein prediction. The predicted domain was searched to find out the related protein structure to be used as a template by the BLAST (Basic Local Alignment Search Tool) (Altschul, S. F et al 1997 and, Altschul et al 1990) program against PDB (Protein Data bank). Sequence that showed maximum identity with high score and less e-value was aligned and was used as a reference structure to build a 3D model for Tyrosine Kinase protein. The co-ordinates for the structurally conserved regions (SCRs) for Tyrosine Kinase protein were assigned from the template using multiple sequence alignment, based on the Needleman-Wunsch algorithm (Needleman, S. B. and Wunsch, C. D. 1970).

\section{Molecular Dynamics}

The structure having the least modeller objective function, obtained from the modeller was improved by molecular dynamics and equilibration methods using NAMD 2.5 software (Kale L et al 1999) using CHARMM27 force field for lipids and proteins (Schlenkrich, M et al 1996) along with the TIP3P model for water (Jorgensen, W. L et al 1983). The energy of the structure was minimized with 1, 00,000 steps. A cutoff of $12 \AA$ (switching function starting at $10 \AA$ ) for van der Waals interactions was assumed. No periodic boundary conditions were included in this study. An integration time step of 2 fs was used, permitting a multiple timestepping algorithm (Grubmuller, $\mathrm{H}$ et al 1991) to be employed in which interactions involving covalent bonds were computed every time step, short-range nonbonded interactions were computed every two time steps, and long-range electrostatic forces were computed every four time steps. The pair list of the nonbonded interaction was recalculated every ten time steps with a pair list distance of $13.5 \AA$. The short-range nonbonded interactions were defined as van der Waals and electrostatics interactions between particles within $12 \AA$. A smoothing function was employed for the van der Waals interactions at a distance of $10 \AA$. CHARMM27 [force-field parameters were used in all simulations in this study. The equilibrated system was simulated for 1 ps with a 500 $\mathrm{kcal} / \mathrm{mol} / \mathrm{A} 2$ restraint on the protein backbone under $1 \mathrm{~atm}$ constant pressure and $310 \mathrm{~K}$ constant temperature (NPT) and the Langevin damping coefficient was set to 5 ps unless otherwise stated. With no harmonic constraints, the simulations ran for $30 \mathrm{~ns}$ in the NPT ensemble using Langevin dynamics at a temperature of 300 K. Pressure was maintained at 1 atm using the Langevin piston method with a piston period of $100 \mathrm{fs}$, a damping time constant of $50 \mathrm{fs}$, and a piston temperature of $300 \mathrm{~K}$. Non-bonded interactions were smoothly switched off from 10 to $12 \mathrm{~A}^{\circ}$. The list of non-bonded interactions was truncated at $14 \mathrm{~A}^{\circ}$.

\section{Structure Validation}

Finally, the structure having the least energy with low RMSD (Root Mean Square Deviation) was used for further studies. In this step, the quality of the initial model was improved.The final structure obtained was analyzed by Ramachandran's map using PROCHECK (Programs to check the Stereo chemical Quality of Protein Structures) (Brunger, A et al 1992) and environment profile using ERRAT graph (Structure Evaluation server) (Laskoswki et al 1993). This model was used for the identification of active site and for docking of the substrate with the enzyme. 


\section{Binding-site analysis}

The Bindig-site of Tyrosine Kinase was identified using CASTP server. A new program, CAST, for automatically locating and measuring protein binding pockets and cavities, is based on precise computational geometry methods, including alpha shape and discrete flow theory. CAST identifies and measures pockets and pocket mouth openings, as well as cavities. The program specifies the atoms lining pockets, pocket openings, and buried cavities; the volume and area of pockets and cavities; and the area and circumference of mouth openings. When the search is complete, the largest site is automatically displayed on the structure. The results can be used to guide the protein -ligand docking experiment.

\section{Molecular docking}

30 analogs were built and optimized with Chemsketch software. Ligands were docked with model Tyrosine Kinase by GOLD software. GOLD is an implementation of multi conformer docking, which implies conformational search of the ligand is carried out. Thereafter all relevant low-energy conformations are rigidly placed in the binding site. The GOLD process uses a series of shape-based filters and default scoring function (based on Gaussian shape fitting) (Diaz et al., 2004).

\section{Results And Discussion}

Homology Modelling of Tyrosine Kinase protein Domain

A high level of sequence identity should guarantee more accurate alignment between the target sequence and template structure.In the results of BLAST search against PDB, only the 1RJB which has a high level of sequence identity with the Human Tyrosine Kinase protein domain. Structurally conserved regions (SCRs) for the model and the template were determined by superimposition of the two structures and multiple sequence alignment.

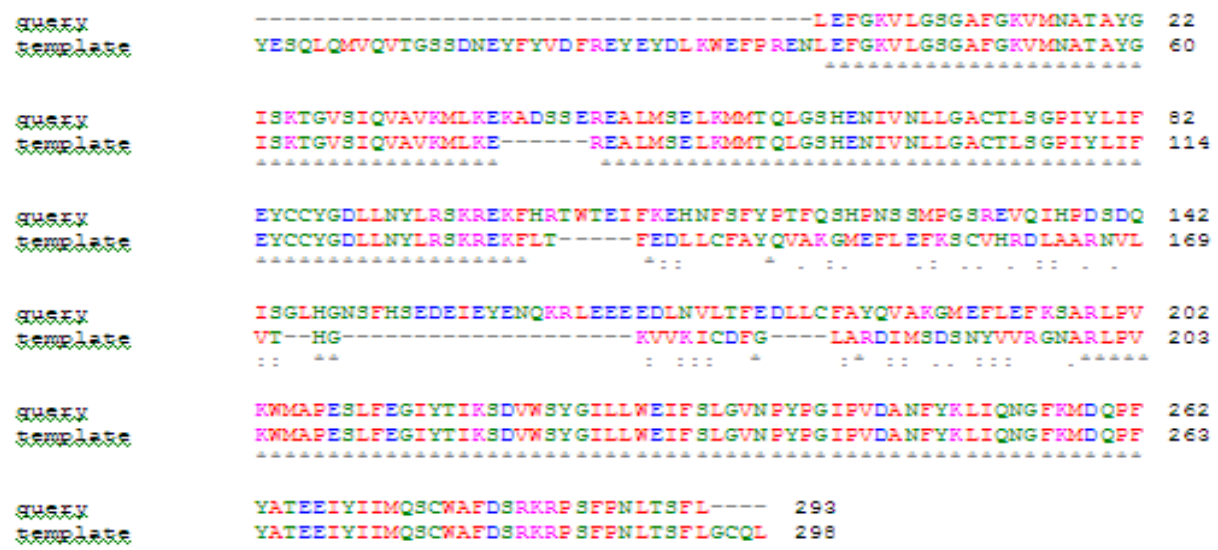

Figure 1: Alignment of Tyrosine kinase with $1 \mathrm{Z3S}$

In the following study, we have chosen $1 \mathrm{Z3S}$ as a reference structure for modeling Human Tyrosine Kinase protein domain. Coordinates from the reference protein (1Z3S) to the SCRs, structurally variable regions (SVRs), N-termini and C-termini were assigned to the target sequence based on the satisfaction of spatial restraints. In the modeller we will get a 20 PDB out of which we select a least energy. The energy unit will be in kilo joule. All side chains of the model protein were set by rotamers. The final stable structure of the Human Tyrosine Kinase protein obtained is shown in Figure 2.

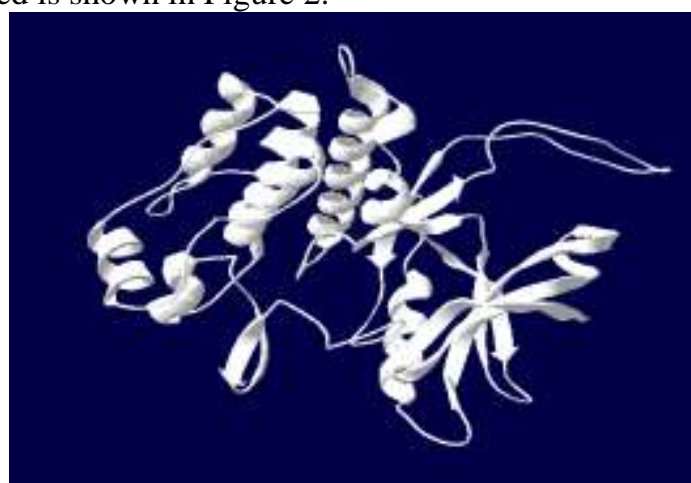

Figure 2: Modeller result 
The structure having the least energy with low RMSD (Root Mean Square Deviation) which was obtained by the NAMD is in Figure 2.

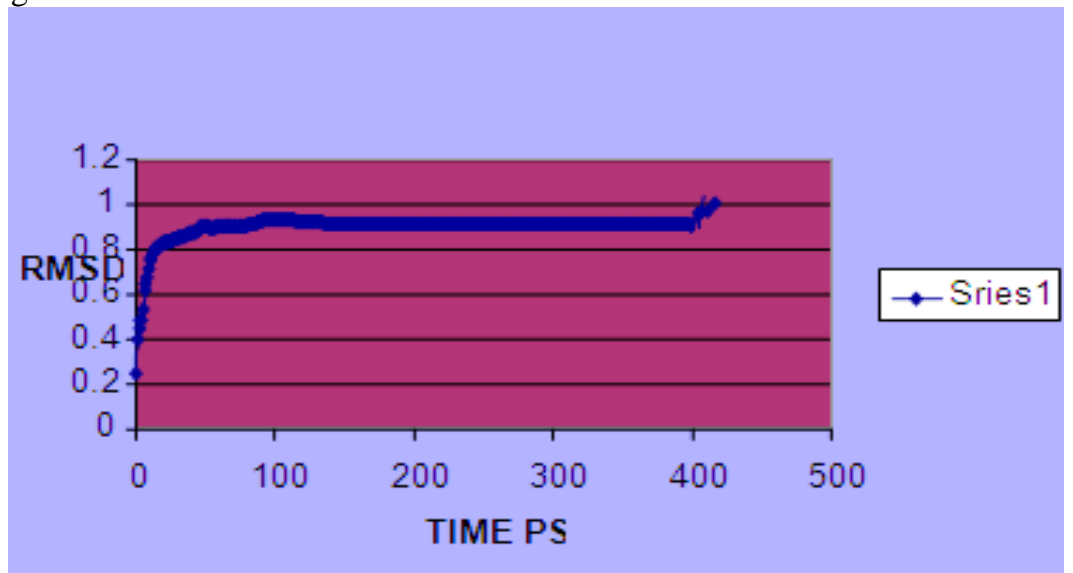

Figure 3: Calculated RMSD graph of molecular dynamics simulations of Human Tyrosine Kinase protein using NAMD software. Time (Ps) was taken in X-axis and RMSD in Y-axis

By the help of SPDBV it is evident that Human Tyrosine Kinase protein domain has 9 helices and 10 sheets. The final structure was further checked by verify3D graph and the results have been shown in Figure 4 .

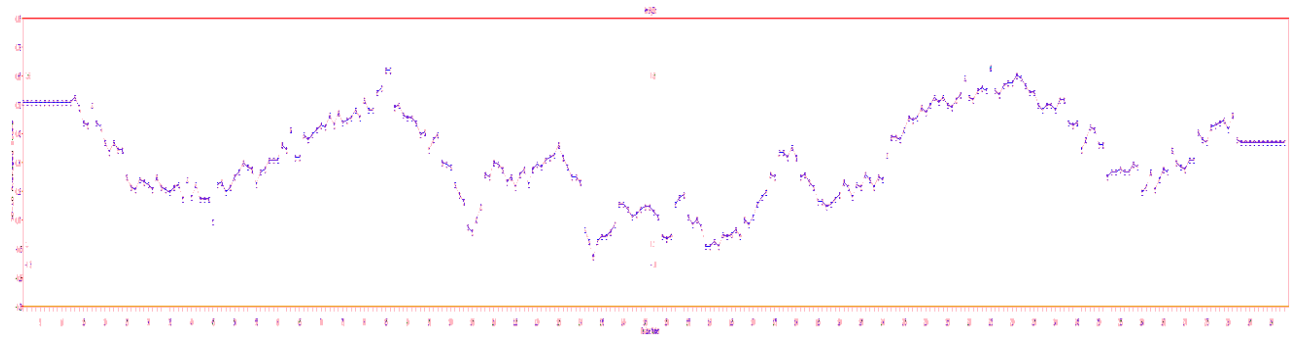

Figure 4: The 3D profiles verified results of Human Tyrosine Kinase Protein model; overall quality score indicates residues are reasonably folded.

\section{Validation of Human Tyrosine Kinase protein Domain}

After the refinement process, validation of the model was carried out using Ramachandran plot calculations computed with the PROCHECK program. The $\Psi$ and $\theta$ distributions of the Ramachandran plots of non-glycine, non-proline residues are summarized in Table I. The RMSD (Root Mean Square deviation) deviation for covalent bonds and covalent angles relative to the standard dictionary of Human Tyrosine KINASE protein was -4.27 and $-0.85 \AA$. Altogether $100 \%$ of the residues of Human Tyrosine protein was in favored and allowed regions. The overall PROCHECK G-factor of Human Tyrosine Kinase protein was -2.32 and verify3D environment profile was good.

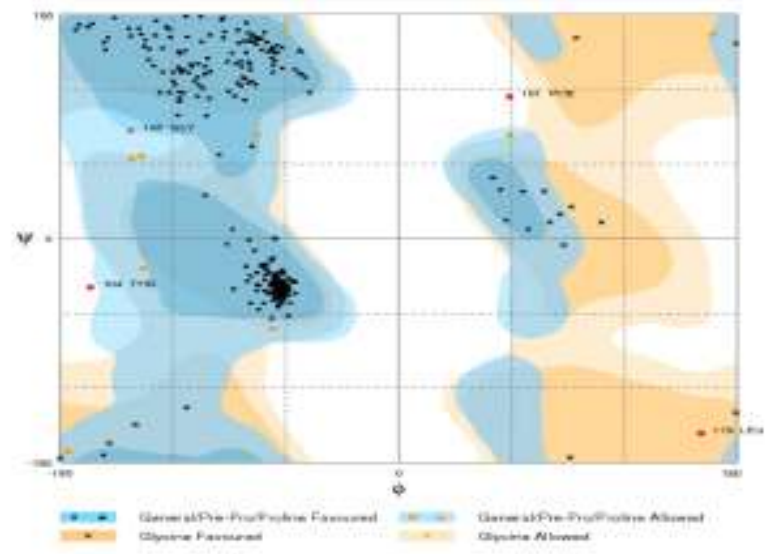

Figure 5: Ramachandran plot 
Table I: \% of residue falling in the core region of the Ramachandran's plot

\begin{tabular}{lc}
\hline$\%$ of residue in most favored regions & 90.0 \\
$\%$ of residue in the additionally allowed zones & 8.9 \\
$\%$ of residue in the generously regions & 1.1 \\
$\%$ of residue in disallowed regions & 0.0 \\
$\%$ of non-glycine and non-proline residues & 100.0
\end{tabular}

\section{Superimposition of 1Z3S with Human Tyrosine kinase protein domain}

The structural superimposition of $1 \mathrm{Z3S}$ template and Human Tyrosine Kinase protein is shown in Figure 6. The weighted root mean square deviation of trace between the template and final refined models $0.42 \mathrm{~A}^{\mathrm{o}}$. This final refined model was used for the identification of active site and for docking of the substrate with the domain Human Tyrosine kinase protein.

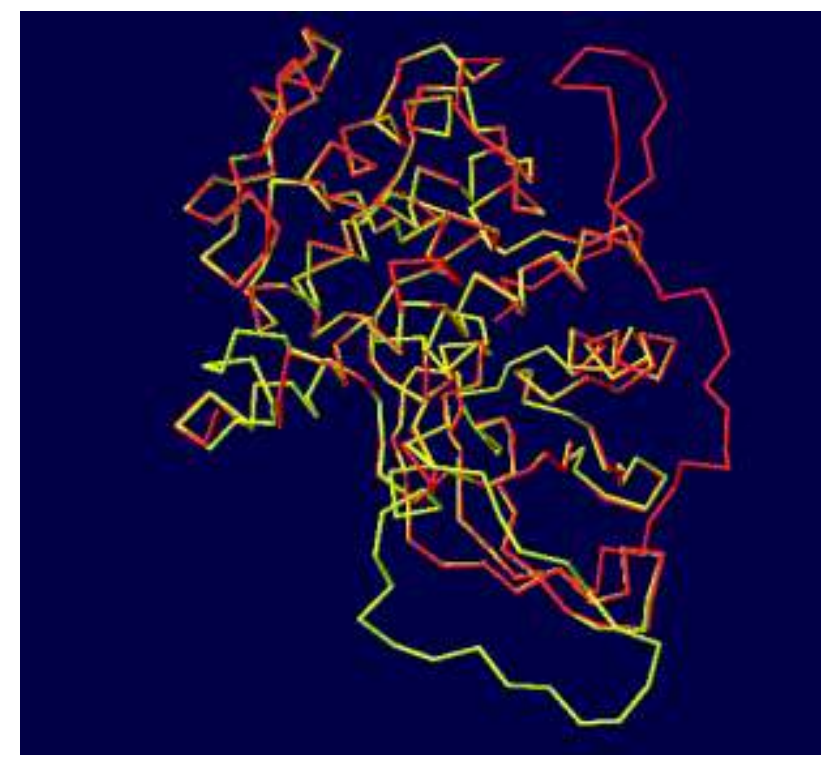

Figure 6: superimposition of $\mathrm{C} \alpha$ trace of Human Tyrosine kinase protein (red colour) and 1Z3S (represented in yellow color).

\section{Activesite Identification}

Active site is obtained using CASTp server, and the location of the site in the 3D structure of Tyrosine Kinase is shown in Fig.7. In fact from the sequence alignment of Tyrosine Kinase with 1Z3S, we know that the residues GLY-21, LEU-22, SER_23, LEU-24, GLU-25, ARG-216, VAL-217, and HSD-220 are conserved. By considering the experimental fact that the active site of $1 \mathrm{Z3S}$ includes all the residues mentioned above, and on other hand, the shape of the site in Tyrosine kinase is similar to that of $1 \mathrm{z} 3 \mathrm{~s}$ binding site. $\mathrm{T}$ Thus in this study the binding site is chosen to dock the analouges.

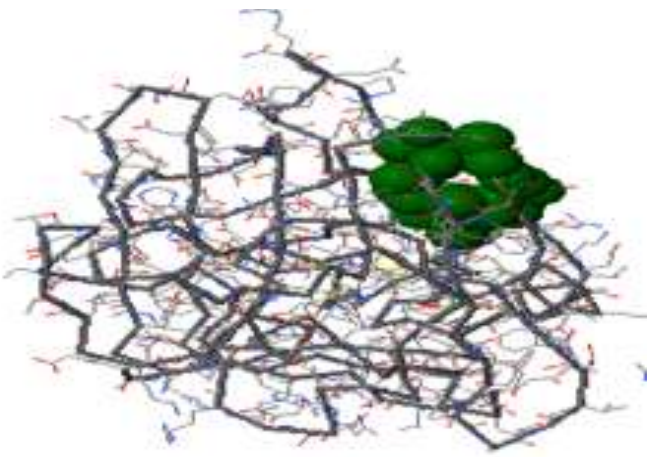

Figure-7: Identification Of Active Site 
<smiles>[R]c1ccc2c(c1)NC(=O)C21SCC(=O)N1Cn1c(-c2ccccc2)nc2ccccc2c1=O</smiles>

Figure-8: STRUCTURE OF 3'-(4-OXO-2-PHENYLQUINAZOLIN-3(4H)-YL)-4'H-SPIRO[INDOLE-3,2'$[1,3]$ THIAZOLIDINE]-2,4'(1H)-DIONE

\section{Docking study}

In the presence of inhibitory results of analogues, new structural features and fictionalization requirements were proposed for the basic scaffold that could increase affinity with Tyrosine Kinase. Among these requirements, the modifications of the group in the structure by a more polar group were expected to increase the activity. These interesting results prompted us to prepare analogues. Here we developed different analogues by replacing with more polar groups than already existing groups, with little change in the properties of analogues represented in Table 1. These were used for docking studies to identify better drug derivative. Docking of inhibitors given in Table 1 (see supplementary material) with Tyrosine Kinase was performed using GOLD 3.0.1, which is based on genetic algorithm (CCDC). The docking procedure includes several steps. First, the protein-ligand complex is generated using the GOLD package without constraints between the ligand and the specific amino acids of the pocket. The algorithm exhaustively searches the entire rotational and translational space of the ligand with respect to the receptors. The flexibility of the ligand is given by dihedral angle variations. The various solutions evaluated by a score, which is equivalent to the absolute value of the total energy of the ligand in the protein environment. Thus docking with the program GOLD version 3.0.1 was employed to locate the appropriate binding orientation and conformation of compounds with Tyrosine Kinase.

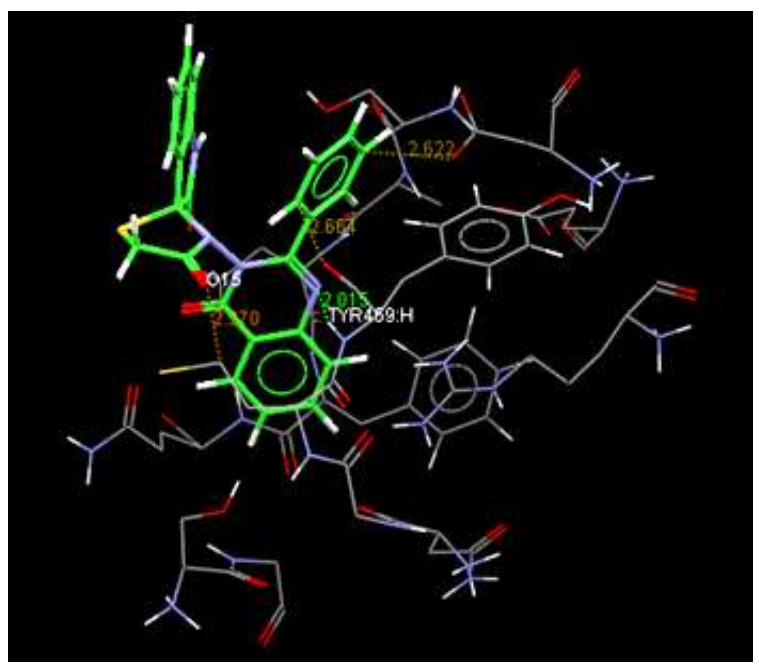



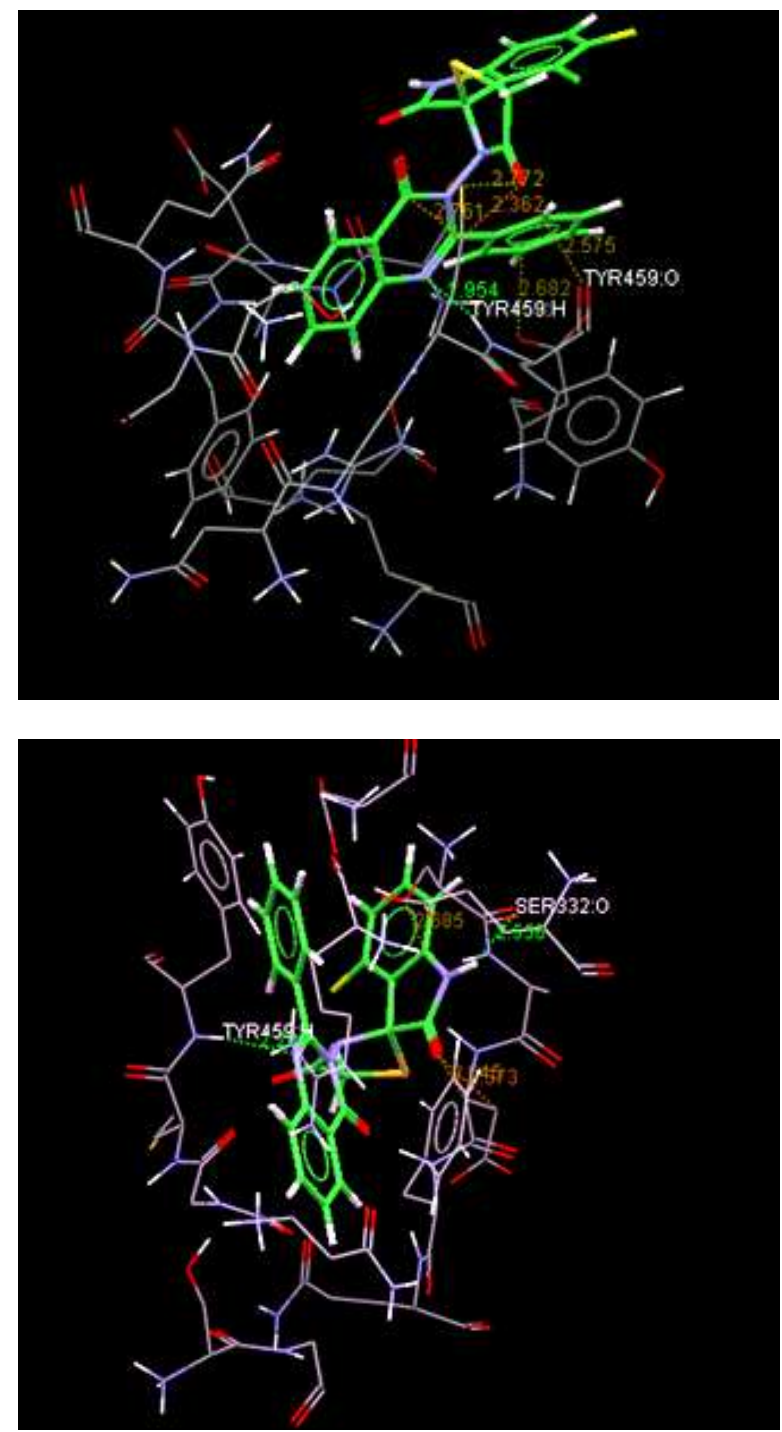

Table 1: Docking scores

\begin{tabular}{cccccc} 
Fitness S(hb_ext) & S(vdw_ext) & \multicolumn{2}{l}{ S(hb_int) } & S(int) & Ligand \\
39.54 & 0.36 & 31.83 & 0.00 & -4.59 & mol1 \\
39.89 & 0.44 & 31.67 & 0.00 & -4.10 & mol10 \\
40.27 & 1.32 & 31.21 & 0.00 & -3.97 & mol11 \\
33.16 & 0.34 & 28.47 & 0.00 & -6.34 & mol12 \\
43.08 & 0.28 & 34.46 & 0.00 & -4.57 & mol13 \\
35.81 & 2.12 & 29.57 & 0.00 & -6.97 & mol14 \\
33.75 & 1.65 & 28.96 & 0.00 & -7.73 & mol15 \\
38.55 & 1.66 & 30.02 & 0.00 & -4.39 & mol16 \\
39.92 & 1.78 & 30.10 & 0.00 & -3.24 & mol17 \\
38.55 & 0.44 & 30.87 & 0.00 & -4.34 & mol18 \\
39.61 & 1.53 & 32.93 & 0.00 & -7.19 & mol19 \\
41.03 & 2.44 & 30.58 & 0.00 & -3.46 & mol2 \\
39.77 & 2.40 & 30.06 & 0.00 & -3.97 & mol20
\end{tabular}




$\begin{array}{cccccc}-25.64 & 0.32 & 31.32 & 0.00 & -69.03 & \mathrm{~mol} 21 \\ 32.22 & 0.29 & 34.41 & 0.00 & -15.38 & \mathrm{~mol} 22 \\ 34.33 & 0.44 & 30.30 & 0.00 & -7.76 & \mathrm{~mol} 23 \\ 42.09 & 2.00 & 37.60 & 0.00 & -11.61 & \mathrm{~mol} 24 \\ 36.50 & 0.38 & 33.82 & 0.00 & -10.38 & \mathrm{~mol} 25 \\ 18.70 & 2.13 & 29.43 & 0.00 & -23.90 & \mathrm{~mol} 26 \\ 41.13 & 2.00 & 38.29 & 0.00 & -13.52 & \mathrm{~mol} 27 \\ 37.12 & 0.94 & 32.79 & 0.00 & -8.91 & \mathrm{~mol} 28 \\ 32.34 & 1.15 & 30.03 & 0.00 & -10.10 & \mathrm{~mol} 29 \\ 39.89 & 2.03 & 30.84 & 0.00 & -4.55 & \mathrm{~mol} 3 \\ 40.54 & 0.55 & 36.31 & 0.00 & -9.94 & \mathrm{~mol} 30 \\ 40.48 & 2.04 & 30.81 & 0.00 & -3.92 & \mathrm{~mol} 4 \\ 36.25 & 0.37 & 35.42 & 0.00 & -12.81 & \mathrm{~mol} 5 \\ 31.12 & 0.29 & 29.34 & 0.00 & -9.51 & \mathrm{~mol} 6 \\ 39.55 & 1.50 & 30.93 & 0.00 & -4.47 & \mathrm{~mol} 7 \\ 38.43 & 0.54 & 30.67 & 0.00 & -4.28 & \mathrm{~mol} 8 \\ 39.20 & 1.67 & 31.39 & 0.00 & -5.63 & \mathrm{~mol} 9\end{array}$

\section{Basic Guidelines for Microwave Organic Chemistry Applications.}

\section{Introduction:}

The main advantages of microwave assisted organic synthesis are:

a. Faster reaction: the microwave can use higher temperatures than conventional heating system, and consequently the reactions are completed in few minutes instead of hours

b. Better yield and higher purity: less formation of side product are observed using microwave irradiation, and the product is recovered in higher yield. Consequently, also the purification step is faster and easier.

c. Easy scale-up: its technology and large range of reactor vessels, allows scale-up from few milliliters to one liter without changing reaction parameters.

d. Reproducibility: the patented microwave diffuser for homogeneous microwave irradiation inside the cavity and precise control of reaction parameters, such as temperature, pressure and power, always reproduces the same reaction conditions. It is very simple to save and use an optimized synthesis method.

e. Easy to use: all the reactors and software are very easy to use and all reactions can be easily moved from conventional to microwave heating.

\section{How To Convert A Conventional Reaction Into A Microwave Reaction}

When the reaction is performed the first time under microwave irradiation, run the reaction in small scale, slowly increasing the temperature.

The parameters that are needed to be defined are: -

- Solvent

- Temperature-time

- Vessel

- Microwave program

Solvent The same solvent that is usually used with conventional heating chemistry can also be used with microwave heating. Solvents interact differently with microwaves, depending on their polarity. Polar solvents (alcohols, DMF, water, ketone, acid) couple well with microwaves and reach high temperatures in a short time. Non-polar solvents (toluene, chloroform, hexane) are transparent to microwaves. Therefore, two situations are possible: 1) non polar solvent, but polar reagents or at least one polar reagent: the reaction mixture is heated by microwave. 2) non polar reaction mixture (both solvent and reagents). (Weflon has to be added in order to heat the mixture.

\section{Temperature-Time}

a) If the reaction has already been performed with conventional heating, take in consideration the standard reaction temperature and time. Based on these two parameters, consider the Arrhenius equation, e.g. 
how the time decreases when the temperature increases. This law defines that every ten degrees that the temperature increases, the time of the reaction is halved.

For example, if a reaction is run in $\mathrm{EtOH}$ at $80^{\circ} \mathrm{C}$ for 8 hours and the Arrhenius law is applied, the time is reduced in accordance to the table below

\begin{tabular}{|c|c|}
\hline $\begin{array}{c}\text { Temperature } \\
\left({ }^{\circ} \mathrm{C}\right)\end{array}$ & Time \\
\hline 80 & $8 \mathrm{~h}$ \\
\hline 90 & $4 \mathrm{~h}$ \\
\hline 100 & $2 \mathrm{~h}$ \\
\hline 110 & $60 \mathrm{~min}$ \\
\hline 120 & $30 \mathrm{~min}$ \\
\hline 130 & $15 \mathrm{~min}$ \\
\hline 140 & $8 \mathrm{~min}$ \\
\hline 150 & $4 \mathrm{~min}$ \\
\hline
\end{tabular}

This simple procedure can be applied to all the reactions. b) If the reaction has never been performed before with conventional or microwave heating, fix the temperature at $30-40^{\circ} \mathrm{C}$ higher than the boiling point of the solvent, and run the reaction for 10 minutes. Then check the obtained reaction mixture

Vessel

All reactors that work with the Micro synthesizer have different:

a) volume limit b) temperature limit c) pressure limit When the target temperature is fixed. Check which vapor pressure is developed from the solvent at the chosen temperature. Based on this value, and on the volume that is needed, decide the appropriate reactor vessel.

\section{How To Optimize The Reaction Conditions}

After the first run of the reactions, there could be four different cases:

1) The reaction is complete (the starting material is not present any more): transfer the mixture in proper glassware and proceed with the usual work-up of the reaction.

2) The reaction starts to work but is not complete (some starting material is still present): - extend the reaction time - increase the temperature (not over the temperature and pressure limit of the vessel).

3) The reaction doesn't work at all: - extend the time - increase the temperature - use more equivalent of one of the starting material or of the catalyst

4) Decomposition of the reagents: - use lower temperature - use short reaction time Note: always remember to check the temperature and pressure limit of the vessel before increasing the temperature.

\section{SYNTHESIS}

Synthetic method development trials was found that not possible of making the thiozolidine moiety on $3-\{[(3 E)$ 2-oxo-1,2-dihydro-3H-indol-3-ylidene]amino\}-2-phenylquinazolin-4(3H)-one derivatives with thioglycolic acid.

\section{SYNTHESIS}

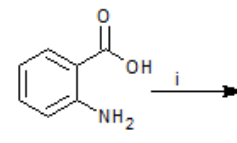<smiles>O=c1oc(-c2ccccc2)nc2ccccc12</smiles>

(l)
SCHEME:

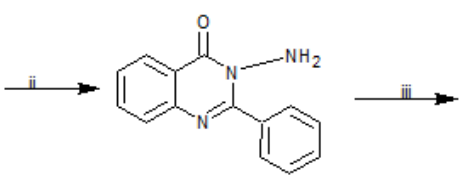

(II)<smiles></smiles>

(III)

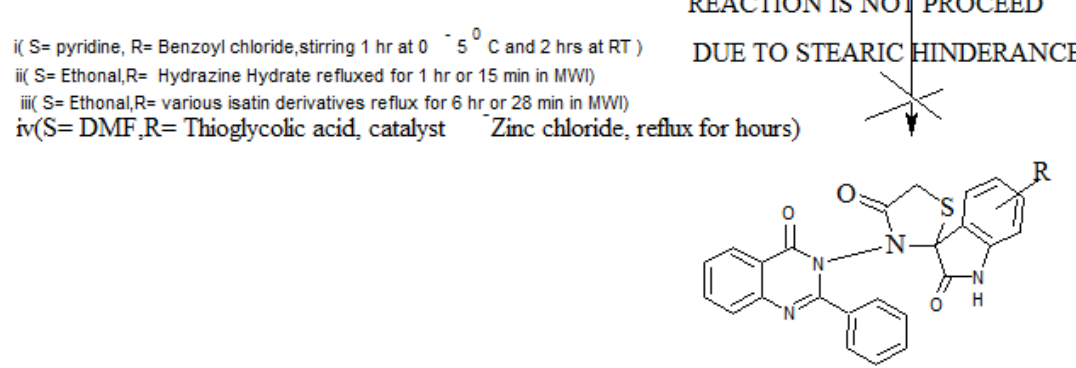


Experimental Proceedure:

Step -I-(2-phenyl-4H-3,1-benzoxazin-4-one)

In to $250 \mathrm{ml}$ of conical flask 1.37 gram of anthranilic acid was dissolved in the $10 \mathrm{ml}$ of pyridine then add $1.4 \mathrm{ml}$ of benzoyl chloride as drop wise while the mixture is stirring and maintain the temperature at 5 degree Celsius for 60 min with continues stirring.

After that stir the reaction mixture for 120 min at room temperature, a solid precipitate will be form, and then precipitate was collected by filtration. Recrystalization by the help of ethanol.

Percentage of yield is $87 \%$

MeltingPoint: $122-125^{\circ} \mathrm{C}$

Boiling Point: $190-193^{\circ} \mathrm{C}$.

Step-II-(3-amino-2-phenylquinazolin-4(3H)-one).

0.01 mole (2-phenyl-4H-3,1-benzoxazin-4-one) of sample was taken in ethanol. To this equimolar quantity of hydrazine hydrate was added and refluxed for 15 minutes under microwave synthesizer or one hour reflux. Then the reaction was cooled. Product so formed as precipitate by filteration, dried and recrystallised using ethanol. Purity of the compound was checked by TLC using hexane and ethyl acetate solvent system in 8:2 ratio. Melting point was found to be 179-182 degree celcius.

Percentage of yield is $92 \%$

\section{Step-III:}

3-\{[(3E)-2-OXO-1,2-DIHYDRO-3H-INDOL-3-YLIDENE]AMINO\}-2-PHENYLQUINAZOLIN-4(3H)-

ONE

0.01 mole of 3-amino-2-phenylquinazolin-4(3H)-one dissolved in ethanol and 0.01 mole of different isatin derivative each time in to the round bottom flask and reflux under microwave synthesizer for 28 minutes or 6 hours for conventional reflux. After completion of the reaction kept a side for cooling the reaction, then forms precipitate which we collect by filtration. Then dry the compound and recrystalized with alcohol.

Percentage of yield is $89 \%$

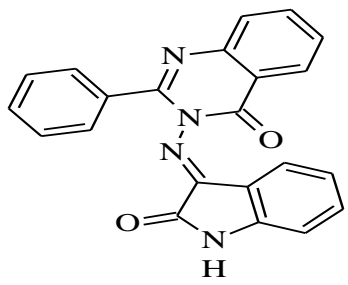

3-\{[(3E)-2-oxo-1,2-dihydro-3H-indol-3-ylidene $]$ amino $\}$-2-phenylquinazolin-4(3H)-one

\section{Step IV:}

0.001 mole of 3-\{[(3E)-2-oxo-1,2-dihydro-3H-indol-3-ylidene $]$ amino $\}$-2-phenylquinazolin-4(3H)-one soluble in $10 \mathrm{ml}$ of DMF / T.E.A / Ethanol / Methanol /THF/ toluene/ pyridine, then 0.001 mole of thioglycolic acid is added, to this solvent added catalytic amount of anhydrous zinc chloride. refluxed for hours together by changing the concentrations. Reaction is not proceeded why because stearic hindrance which is having on both sides of nitrogen atom.

\section{Conclusion}

HUMAN Tyrosine Kinase Protein is one of the important proteins in Blood cancer. In this work, we have constructed a 3D model of Human Tyrosine Kinase protein domain, from human using the MODELLER software and obtained a refined model after energy minimization. The final refined model was further assessed by ERRAT \& PROCHECK program, and the results show that this model is reliable. The interaction between the domain and the inhibitors proposed in this study are useful for understanding the potential mechanism of domain and the inhibitor binding. The hydrogen bonds play important role for the structure and function of biological molecule in this study, and we found that GSN235, AsN257, and ILE289 of Tyrosine Kinase are important for strong hydrogen bonding interaction with these inhibitors. To the best of our knowledge GSN235, AsN257, and ILE289 are conserved in the domain and may be important for structural integrity or maintaining the hydrophobicity of the inhibitor-binding pocket. After synthetic method development trials was found that not possible of making the thiozolidine moiety on $3-\{[(3 E)$-2-oxo-1,2-dihydro-3H-indol-3-ylidene $]$ amino $\}-2$ phenylquinazolin-4(3H)-one derivatives with thioglycolic acid. 


\section{Acknowledgements}

The authors gratefully acknowledge the UGC and AICTE for providing the provision for research in JNTUH, Hyderabad. Special Thanks to Centre for Pharmaceutical Sciences, Institute of Science and Technology, JNTUH, Kukatpally, Hyd-85 for providing infrastructure facilities to carry out this research work.

\section{References}

[1]. Abu-Duhier FM, Goodeve AAC, Wilson GA, Care RS,Peake IR, Reilly JT9(2001): identification of novel FLT-3 Asp835 mutations in adult acute myeloid leukemia. Br J Haematol, 113:983-988

[2]. Altschul, S. F., Gish, W., Miller, W., Myers, E. W. and Lipman, D. J. A basic local alignment search tool. J. Mol. Biol. 215, (1990) 403-410.

[3]. Altschul, S. F., Madden, T. L., Schaffer, A. A., Zhang, J., Zhang, Z., Miller, W. and Lipman, D. J. (1997). Gapped BLAST and PSIBLAST: a new generation of protein database search programs. Nucleic Acids Res. 50, 3389-3402.

[4]. Brig F, Courcoul M, Rosnet O,Bardin F, Pebusque MJ, Marchetto S, Tabilo A, Mannoni P, Birnbaum D (1992): Expression of the FMS/KIT-like gene FLT3 in hguman acute leukemias of the myeloid and lymphoid lineages.Blood,80:2584-2593.

[5]. Brunger, A. 1992. X-PLOR, Version 3.1: A System for X-Ray Crystallography and NMR. Yale University, New Haven, CT.

[6]. Carow CE, Levenstein M, Kaufmann SH, Chen J, Amin S, Rockwell P, Witte L, Borowitz MJ, Civin Ci, Small D (1996): Expression of the hematopoitic growth factor receptor FLT3(STK-1/Flk2)) in human leukemias. Blood, 87:1089-1096.

[7]. Drexler HG (1996): Expression of FLT-3 receptor and response to FLT3 ligand by leukemic cells. Leukemia,10:588-599

[8]. Gillaland DG, Griffin JD (2002): The role of FLT3 in hematopoiesis and leukemia.Blood 100:1532-1542.

[9]. Grubmuller, H., H. Heller, A. Windemuth, and K. Schulten. (1991). Generalized Verlet algorithm for efficient molecular dynamics simulations with long-range interactions. Mol. Sim. 6:121-142.

[10]. Grundler R, Miething C, Thiede C, Dusyter J (2005): FLT3-ITD and tyrosine kinase domain mutants induce 2distinct phenotypes in a murine bone marrow transplantation model. Blood, 105:4792-4799.

[11]. Jorgensen, W. L., J. Chandresekhar, J. D. Madura, R. W. Impey, and M. L.Klein. 1983. Comparison of simple potential functions for simulating liquid water. J. Chem. Phys. 79:926-935.

[12]. Kale L, Skeel R, Bhandarkar M, Brunner R, Gursoy A, Krawetz N, Phillips J, Shinozaki A, Varadarajan K, Schulten K: NAMD2: Greater scalability for parallel molecular dynamics.J Comput Phys 1999, 151:283.

[13]. Klo H, Naoe T, Nakano Y, Yokota S,Minami S, Miyawaki S, Asou N, Kuriyama K, Jinnai I, Shimazaki C, et al (1999) :Prognostic implications of FLT3 and N-RAS gene mutations in acute myeloid leukemia. Blood, 93, 3074-3080.

[14]. Laskoswki, R. A., MacArthur, M. W., Moss, D. S. and Thorton, J. M. (1993). PROCHECK: a program to check the stereochemical quality of protein structures. J. Appl. Cryst. 26, 283-291.

[15]. Li L, Piloto O, Nguyen HB, Greenberg K, Takamiya K, Racke F, Huso D, Small D (2008): Knock-in of internal tandem duplication mutation into murine FLT3 confers myeloproliferative disease in amouse model. Blood, 111:3849-3858.

[16]. Meierhoff G, Dehmel U,Gruss HJ,Rosnet O, Birnbaum D,quentmeier H,Dirks W, Drexler HG (1995): Eexpression of FLT3 receptor and FLT3-ligand in human leukemia-lymphoma cell lines.Leukemia,9:1368-1372.

[17]. Meshinchi S, Stirwalt DL, Alonzo TA, Zhang Q, Sweetser DA, Woods WG, Bernstein ID,Arced RJ, Radich JP (2003): Activating mutations of RTK/ras signal transduction pathway in pediatric acute myeloid leukemia. Blood, 102:1474-1479.

[18]. Nakao M,Yokota S, Lwai T, Kaneko H, Horiike S, Kashima K, Sonoda Y,Fujimoto T, Misawa S(1996), Internal tandem duplication of the flt3 gene found in acute myeloid leukemia. Leukemia 10:1911-1918.

[19]. Needleman, S. B. and Wunsch, C. D. (1970). A general method applicable to the search for similarities in the amino acid sequence of two proteins. J. Mol. Biol. 48, 443-453.

[20]. Schlenkrich, M., J. Brickmann, A. D. MacKerell, Jr., and M. Karplus. (1996). Empirical potential energy function for phospholipids: criteria for parameter optimization and applications. In Biological Membranes: A Molecular Perspective from Computation and Experiment. K. M. Merz and B. Roux, editors. Birkhauser, Boston, MA. 31-81.

[21]. Shih LY, Lin TL, Wang PN, Wu JH, Dunn P, KUo MC, Huang CF (2004): internal tandem duplication of fms-like tyrosine kinase 3 is associated with poor outcome in patients with myeloysplastic syndrome. Cancer, 101.989-998.

[22]. Small D. (2006) FLT3 mutations: biolgy and treatment. Hematology am soc Hematol Edu program, $178-184$.

[23]. Southgate,R. Contemp. Org. Synth. 1994, 1, 417-431.

[24]. Broccolo, F.; Carnally, G.; Caltabiano, G.; Cocuzza, C.E.A.; Fortuna, C.; Galletti, G.; Giacomini, P.D.; Musumarra, G.; Musumeci, R.; Quitavalla, J. Med. Chem. 2006, 49, 2804-2811.

[25]. Alcaide, B.; Almendros, P.; Aragoncillo, C. Chem. Rev. 2007, 107, 4437-4492. 